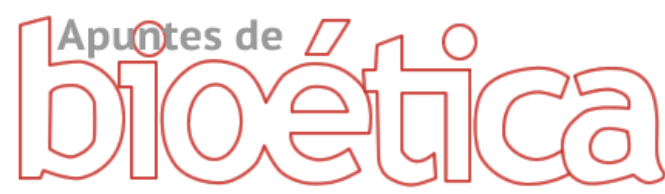

https://doi.org/10.35383/apuntes.v3i1.399

\title{
Presencia, autoconciencia y autocuidado de los profesionales que trabajan con el sufrimiento
}

\author{
Benito E. ${ }^{1}$, Rivera-Rivera P. ${ }^{2}$, Yaeguer J. ${ }^{3}$, Specos M. ${ }^{4}$
}

INFORMACIÓN DEL ARTÍCULO RESUMEN

Historia del artículo:

Recibido el 25 de mayo 2020

Aceptado el 28 de julio 2020

\author{
Palabras claves: \\ Autocuidado \\ Autoconciencia \\ Personal Sanitario \\ Satisfacción Laboral \\ Burn out
}

El trabajo en el entorno sanitario expone frecuentemente a los profesionales a experiencias de intensidad emocional, que emergen del contacto con la vulnerabilidad, las pérdidas y el sufrimiento de pacientes y familiares, lo que supone una elevada exposición al estrés.

Los avances en neurobiología y la mejor comprensión de la neurofisiología de la relación terapéutica, permiten entender cómo podemos, no solo cuidarnos mejor, sino como dotarnos de recursos para transformar constructivamente la experiencia del que sufre y del que acompaña.

La compresión del rol de la autoconciencia, la presencia y el autocuidado y sus fundamentos neurobiológicos, nos permiten elaborar propuestas para prevenir el riesgo de burn out y otros problemas de salud mental muy prevalentes entre los profesionales.

El artículo revisa las bases de la relación de ayuda, los riesgos y oportunidades de nuestro trabajo, las relaciones entre empatía, autoconciencia y compasión; el balance emocional necesario para trabajar en este entorno y la autoconciencia como plataforma de la presencia y de la regulación emocional.

Se ofrecen algunas prácticas útiles para el autocuidado, incluyendo el trabajo en equipo como factor de protección. $Y$ finalmente se propone un compromiso del profesional con su autocuidado.

\footnotetext{
${ }^{1}$ PhD Internal Medicine. Doctor en Medicina, Experto en Cuidados Paliativos, Profesor Invitado UCM. Colegio Oficial de Médicos de las Islas Baleares: Palma de Mallorca, Islas Baleares, España. Email: benitoenric@gmail.com ORCID: https://orcid.org/0000-0002-0489-4668

${ }^{2}$ Máster en Psicooncología y Cuidados Paliativos, Máster en Neuropsicología, Psicóloga General Sanitaria, y Doctoranda en Biotecnología. Medicina y Ciencias Biosanitarias de la Universidad Francisco de Vitoria, Madrid, España. Email: paolariverarivera@gmail.com. ORCID: https://orcid.org/0000-0002-7157-7785

${ }^{3}$ Médico especialista en Medicina Interna y Paliativista. Asesor ministerio de Salud de Chile del Programa de Cuidados Paliativos. Presidente Sociedad Medicina del Dolor y Cuidados Paliativos MEDOPAL Chile, Gobierno de Chile Ministerio de Salud: Santiago, Metropolitana, Santiago de Chile, Chile. Correo: pyaeger@gmail.com ORCID: https://orcid.org/0000-0002-6152-0275

${ }^{4}$ Máster en Dirección y Gestión de Instituciones Educativas. Especialista en Educación de Adultos. Licenciada en Ciencias de la Educación. Directora de Estudios de Pallium Latinoamérica. Hospice Buen Samaritano, Buenos Aires, Argentina. Homemarce@yahoo.com.ar ORCID: https://orcid.org/0000-0001-7344-6431
} 


\title{
Presence, self awareness and self care in professionals working with suffering
}

ABSTRACT

\section{Keywords:}

Self-care

Self-awareness

Clinicians

Work Satisfaction

Burn out

\begin{abstract}
Professionals working in a sanitary environment are often exposed to high emotional experiences that rise from the contact with vulnerability, loss, and suffering of patients and relatives, leading to a high exposition to stress.

Advances in neurobiology and a higher knowledge of therapeutic relationships allow a better understanding of how we can look after ourselves better and provides resources to transform the experience of sufferers and companions.

Understanding the role of self-awareness, presence and self-care and their neurobiological fundamentals allow the development of proposals to prevent the risk of burnout and other prevalent mental health problems among professionals.

This work reviews the basis of help relationship, the risks and chances of our work, the relationship between empathy, self-awareness and compassion, as well as the emotional balance needed to work in this environment and self-awareness as a platform of presence and emotional regulation.

Some useful practices for selfcare are presented, including teamwork as a protection factor. Finally, a commitment of professionals with their self-care is proposed.
\end{abstract}

\section{Introducción}

En la medida que trabajamos desde una conciencia despierta, presente, ecuánime y compasiva, podremos establecer relaciones sanadoras para los pacientes y vivir experiencias transformadoras para ambos.

Así el autocuidado deja de ser algo "que hacer" al salir del trabajo, para convertirse en una forma de ser, estar y experimentar en él mismo.

El trabajo en el entorno sanitario expone frecuentemente a los profesionales a experiencias de intensidad emocional, que emergen del contacto con la vulnerabilidad, las pérdidas, y el sufrimiento de pacientes $y$ familiares.

En esta atmósfera de impacto, generalmente trabajamos bajo presión asistencial, tomando decisiones a menudo con incertidumbre y responsabilidad, y frecuentemente sin formación específica, lo que supone una elevada exposición al estrés.

El paradigma biomédico que domina en el modelo sanitario, apenas integra la dimensión subjetiva de la persona, y las emociones y el sufrimiento no suelen ser considerados en la formación académica, a pesar de que impregnan todo el quehacer y la relación médico-paciente.

La falta de formación en gestión emocional y de autocuidado del profesional, ha llevado a un crecimiento exponencial de los problemas de burnout y de salud mental de los sanitarios, que trabajan sin recursos específicos para gestionar estas situaciones. Esta realidad tiene su impacto económico en el aumento del absentismo laboral y la sobrecarga del sistema sanitario.

Algunos profesionales de cuidados paliativos, enfrentados cotidianamente al 
proceso de morir y al sufrimiento que este conlleva, se han visto obligados a explorar cómo enfrentar y prevenir el desgaste emocional y promover su propia resiliencia.

Los avances en neurobiología y la mejor comprensión de la neurofisiología de la relación terapéutica, permiten entender cómo podemos, no solo cuidarnos mejor, sino como dotarnos de recursos para transformar constructivamente la experiencia del que sufre y del que acompaña.

En la actualidad, la pandemia hace que el sufrimiento sea más visible y colectivo afectando a muchos más profesionales. Por lo que parece oportuno compartir conocimiento y estrategias para ayudar a proteger al ser humano sensible y vulnerable que somos, los profesionales de la salud.

Aunque hay diversos factores externos o del entorno que influyen en el desgaste profesional, nos centraremos en los que dependen exclusivamente del profesional sanitario y que, por ello, se pueden modificar o intentar mejorar.

Tabla 1. Algunas ideas fundamentales:

- Ayudar a otros nos pone en contacto directo con la vida de otras personas. Nuestra condición humana nos conecta.

- Nuestra actitud compasiva de ayudar a las personas que atendemos es fuente de satisfacción, aunque también tiene sus riesgos.

- La enfermedad, el deterioro, las pérdidas y el sufrimiento son percibidas por el paciente como amenaza, distrés y malestar emocional. El profesional, desde la conexión terapéutica, percibe las emociones del paciente y en ocasiones pueden "contagiarlo" y afectarle.
- La mayoría de los profesionales han sido autodidactas en el aprendizaje de cómo enfrentarse con el sufrimiento, sin huir ni contagiarse.

- La comprensión académica de la fisiología de la relación de ayuda, el impacto de las emociones y la pedagogía de cómo gestionarlas son bastante recientes y aún no forman parte de la formación habitual de las profesiones sanitarias.

\section{- Conocer los fundamentos} neuropsicológicos de la relación de ayuda, el concepto de presencia terapéutica y el rol de la autoconciencia, permiten entender la necesidad de cultivar la capacidad de mantener la ecuanimidad y compasión en el entorno del sufrimiento.

- La calidad de nuestra presencia determina el éxito de la relación terapéutica y desde esta perspectiva, el autocuidado del profesional es un imperativo ético.

- El autocuidado no es una práctica que se realiza al salir del trabajo para "desconectar", sino una forma de estar presente $y$ consciente, aprovechando la riqueza experiencial de la relación de ayuda.

\section{Para desarrollar estas ideas intentaremos contestar a estas preguntas:}

- ¿Porque trabajar con las personas que sufren supone para los profesionales un riesgo de "contagio"?

- ¿Qué sabemos de los mecanismos neurofisiológicos y psicológicos que regulan las relaciones humanas, y como explican los riesgos y oportunidades de la relación de ayuda?

- ¿Cuáles son las actitudes para atender constructivamente el sufrimiento sin huir ni "contagiarnos"? 
- ¿Cómo podemos cultivar nuestra capacidad de ayuda sin exponernos al desgaste profesional y aprendiendo de cada encuentro?

\section{Para responder a estos interrogantes vamos a explorar:}

1. Las bases de la relación de ayuda.

2. Riesgos y oportunidades de nuestro trabajo: Estrés secundario o vicario y la satisfacción por compasión.

3. La empatía, autoconciencia y compasión.

4. El balance emocional necesario para trabajar en este entorno.

5. La autoconciencia como plataforma de la presencia y de la regulación emocional.

6. ¿Qué prácticas son útiles para el autocuidado?

7. El trabajo en equipo como factor de protección.

8. El necesario compromiso del profesional con su autocuidado

Metodología. Para lo cual, nos basamos en la experiencia clínica e investigadora del grupo de trabajo denominado Foro Ibero Americano de Espiritualidad en Cuidados Paliativos, del que formamos parte, con apoyo en la bibliografía específica y seleccionada, que aporta información relevante sobre los temas que se plantean, siendo estos: la relación terapéutica, la gestión de las emociones, la presencia terapéutica, autoconciencia y autocuidado, que ha contribuido en la construcción de la propuesta que se presenta.

\section{Bases de la relación de ayuda}

La asistencia sanitaria se cimenta en la relación que se establece entre los diferentes profesionales, el paciente y su familia. La buena práctica y los resultados de ella dependen en gran medida, de la calidad de esta relación terapéutica que pueda construirse.

La conexión que se establece entre el paciente y su familia con los profesionales sanitarios es un territorio en el que son frecuentes las emociones, que a menudo se experimentan de forma intensa. Aquí el profesional debe atender y facilitar la adaptación del paciente a su situación, evitando formar parte del problema, es decir manteniendo su ecuanimidad y su balance emocional.

En medicina clásicamente se ha considerado que la capacidad de mantener la serenidad y el equilibrio emocional en la relación terapéutica es una "virtud" difícil de adquirir y casi imposible de enseñar.

Así Wiliam Osler uno de los padres de la medicina interna, en un discurso a los estudiantes de la Universidad de Pensilvania en 1889 que tituló "Aequanimitas" (Sir William Osler, 2004) les hablaba de la imperturbabilidad, la presencia de ánimo, la calma y la claridad de juicio que debe mantener un profesional ante momentos de dificultad o peligro. Decía que esta era una virtud corporal "apreciada por los legos, y el médico que tiene la desgracia de no poseerla, que delata su indecisión y preocupación, y que muestra que está nervioso y aturullado en las urgencias ordinarias, rápidamente pierde la confianza de sus pacientes".

Otro texto clásico, de medicina interna conocido como "el Harrison" (Whintrobe, Thorn, Adams y Bennett, 1973) hablando de la relación terapéutica nos recuerda que:

El médico no logrará una comprensión profunda de los problemas psicológicos del paciente, a menos que aprenda a reconocer claramente y a dominar sus propios problemas emocionales. Respecto a las relaciones más personales con el 
paciente y a la comprensión y capacidad para profundizar en su conducta $y$ motivaciones, no existe preparación ni adiestramiento alguno capaz de reemplazar al talento intuitivo $y$ al juicio maduro.

Aunque ambos textos apuntaban aspectos cruciales de la relación terapéutica, como la necesidad de gestionar las emociones, de mantener la ecuanimidad y de reconocer que esta es una "virtud corporal", se perciben como aspectos difíciles de transmitir y de enseñar.

\section{Riesgos y oportunidades de nuestro trabajo. Estrés secundario o vicario y la satisfacción por compasión}

Desde que se escribieron los textos citados en el punto anterior, hemos aprendido nuevos aspectos de la relación de ayuda. El estudio y la atención de los enormes daños psicológicos y morales que afectaban a los veteranos de la guerra de Vietnam, generó conocimiento de nuevas patologías como el síndrome de estrés post traumático y el estrés vicario o secundario.

Posteriormente se reconoció como síndrome de fatiga emocional que surge especialmente en aquellos que se dedican a profesiones cuyo objeto de trabajo son las personas.

En el año 1990 McCann y Pearlman dieron a conocer el concepto de Trauma Indirecto o trauma vicario, que definieron como "la transformación negativa en la experiencia interna del terapeuta, que se produce como resultado del compromiso empático con el material traumático de los clientes" es decir, los efectos acumulativos y transformadores sobre los terapeutas que empatizan con pacientes con sufrimiento (McCann y Pearlman, 1990; Pearlman y Mac Ian, 1995).
Algunos años más tarde entre 1995 y 2002 Figley (1995) identificó una construcción relacionada, el estrés traumático secundario (STS), que describió en términos de "el coste de cuidar el dolor emocional de otros" que lleva a los médicos a abandonar su trabajo con personas traumatizadas. Según Figley, la exposición directa e indirecta a acontecimientos traumáticos puede ser impactante y provocar un conjunto de síntomas similares a los del síndrome de estrés postraumático. Con poca fortuna, más tarde a este concepto le daría otro nombre que se ha hecho popular, pese a su inexactitud, el de "fatiga por compasión". (Debería llamarse fatiga por empatía o simplemente estrés post traumático secundario).

El BO es un síndrome relacionado con el trabajo que se manifiesta como resultado de la interacción entre el profesional y la organización (y no tanto con la relación con los pacientes, una diferencia importante con el trauma indirecto o secundario que veremos más adelante) y se caracteriza por tres dominios centrales de síntomas: 1.- agotamiento físico y emocional, 2.cinismo y despersonalización (sensación de desvinculación), y 3.-bajo sentido de logro profesional (sensación de ineficacia).

La prevalencia del BO entre los profesionales de la salud parece que va en aumento, llegando a adquirir dimensiones preocupantes ya que se alcanzan cifras de entre el 28 y el $70 \%$ según diferentes estudios (Navarro-González et al., 2015; Soler et al., 2008; Shanafelt et al., 2012).

Trabajar en un entorno donde el sufrimiento es intenso requiere algunos recursos y habilidades personales que no se enseñan en las facultades de ciencias de la salud, y hacerlo sin ellas es exponerse al riesgo de enfermar mentalmente.

Al igual que existen estos riesgos para los profesionales expuestos al sufrimiento, se da la 
oportunidad de que la relación de ayuda sea una fuente de satisfacción profesional. Lo que conocemos como el gozo de cuidar o la satisfacción por compasión (Stamm, 2012).

$\begin{array}{crrr}\text { Se ha } & \text { observado cómo terapeutas } \\ \text { ejemplares } & \text { trabajando } & \text { con clientes }\end{array}$
traumatizados experimentaron cambios positivos en su sentido profesional, su significado y espiritualidad, describiendo haber adquirido una visión del mundo ampliada, incluso paradójicamente sentirse enriquecidos, como resultado de experimentar el proceso de sanación de sus pacientes.

Cuando el paciente con trauma, lo elabora y trasciende, se da la oportunidad de lo que se conoce como "crecimiento postraumático" (Calhoun y Tedeschi, 1998; Calhoun y Tedeschi, 1999). Este se caracteriza por cambios positivos en las relaciones interpersonales, sentido de sí mismo o autoconciencia, y una filosofía de la vida subsiguiente con la experiencia directa del acontecimiento traumático que remueve las bases de la propia visión del mundo (Calhoun y Tedeschi, 1998; Tedeschi y Calhoun, 1995). El crecimiento postraumático no es poco corriente y puede darse concurrentemente con secuelas negativas de un trauma.

El término "crecimiento vicario postraumático" describe el fenómeno de crecimiento clínico que resulta de presenciar las secuelas positivas de las experiencias traumáticas de otros. Esto puede incluir, en el clínico, sentimientos de que su propia vida ha sido enriquecida, profundizada, 0 se ha visto potenciada por el testimonio del crecimiento postraumático del paciente 0 del familiar (Harrison y Westwood, 2009). Cuando los pacientes experimentan significado y serenidad con relación a la proximidad de su muerte, esto enriquece las vidas de los clínicos que se implican. Este fenómeno es parecido a las "relaciones sanadoras" que identificaron Mount y sus colegas (Mount et al., 2007). Cicely
Saunders ya lo describió como el legado o la herencia que te dejan los pacientes a los que has percibido cómo han visto transformar su limitada perspectiva y han trascendido el sufrimiento (Saunders, 2011).

Vistos los riesgos y oportunidades, la pregunta que surge es ¿Qué factores determinan que algunos profesionales se contagien del sufrimiento $y$ se quemen, mientras otros aprovechan la relación de ayuda para disfrutar de ella y crecer humana y espiritualmente?

¿Y cuál es el fundamento de este cambio? Empecemos por revisar la relación entre sufrimiento empatía y compasión:

\section{Empatía, autoconciencia y compasión}

La atención al sufrimiento supone establecer un vínculo terapéutico y una conexión con los contenidos internos del paciente. Es decir, supone apertura, conexión y al mismo tiempo regular los contenidos que surgen $y$ aparecen en el encuentro sin huir ni sobre implicarse.

La empatía, nos permite compartir las emociones de los demás, y dependiendo de si somos o no capaces de discernir conscientemente, el origen de esta emoción (el otro/yo) puede conducir a la angustia empática, una forma inadaptada de resonancia empática, o a la compasión, un sentimiento de calidez y preocupación por los demás.

Singer y Klimecki (2014) se refiere a la empatía como una capacidad general para resonar con los estados emocionales de los demás, sean estos positivos o negativos. Dentro de este marco general, distingue dos componentes, compasión y distrés empático, como formas de responder al sufrimiento de los demás. La respuesta compasiva, por un lado, se basa en sentimientos positivos orientados hacia 
el otro y en la activación de la motivación y el comportamiento prosocial, mientras que el distrés empático, produce sentimientos negativos y se asocia con la evitación o huida (Batson, 2009; Eisenberg y Eggum, 2009). La compasión es "sentir por" y el distrés empático es "sentir con" el otro, o compartir el sufrimiento del otro (Singer y Klimecki, 2014).

En esta línea Ashar et al. (2017) se refiere a cuidado empático y distrés empático respectivamente, donde el primero conduce a un funcionamiento (emocional) sostenido y el segundo puede causar burnout entre los profesionales (Caroe, 2012).

En la búsqueda de los factores que determinan una conexión eficiente $y$ constructiva, sin riesgo para el profesional veamos algunos avances en neurociencia:

Uno de ellos es el concepto de la neuroplasticidad, es decir nuestra capacidad de modular y transformar nuestro cerebro a través de la experiencia y/o entrenamiento. Esto abre la posibilidad de "reformular nuestros circuitos neuronales" o reescribir los relatos personales a través de la experiencia, que incluye la relación terapéutica. Así podemos entender cómo, a través de la experiencia relacional que se establece en un ambiente terapéutico, partiendo del distrés o del sufrimiento se puede reconstruir y sanar el propio relato vital.

Otro factor es la descripción de las neuronas espejo, la comprobación de que nuestro sistema nervioso, a diferencia de lo que se creía, es un sistema abierto que interactúa y se modifica según la realidad del entorno con el que nos relacionamos.

La aparición de la resonancia magnética funcional, técnica que permite la evaluación funcional de regiones específicas del cerebro durante los procesos cognitivos o emocionales, ha permitido generar conocimiento que nos lleva a entender mejor las bases neurobiológicas de la empatía y la compasión, del contagio y la regulación emocional, tan importantes en la relación terapéutica.

Y quizás uno de los avances y aportaciones más interesantes ha sido la Teoría Polivagal propuesta por Stephen Porges (Porges, 1995; Porges, 2009) que enmarca la relación terapéutica en el contexto de la conexión social que establecemos a través de la neurocepción.

\section{El balance emocional necesario para trabajar en este entorno}

El profesional sanitario debe manejar una gran cantidad de información, referida no sólo a los aspectos biológicos de la enfermedad, a los resultados de las pruebas complementarias, o a los síntomas y tratamiento del paciente, sino además a las emociones del paciente y la familia, las suyas propias, y las que surgen en el encuentro clínico, en el que además debe tomar decisiones, a veces en medio de elevada incertidumbre y presión.

Además, el profesional debe informar de forma sensible sobre aspectos pronósticos y evaluar la capacidad del paciente para recibir información negativa etc., todo ello en un espacio reducido de tiempo y sometido a la presión de que cada problema es único y a veces vital para cada paciente.

Para ello necesita de un nivel elevado de conciencia, para hacerlo con flexibilidad $y$ eficiencia, sin perder la perspectiva realista y compasiva y sin quedar atrapado en las situaciones complejas y a veces impactantes que vive.

La capacidad para gestionar las propias emociones es crucial para una práctica terapéutica eficiente y saludable (Whintrobe, Thorn, Adams, Bennett, 1973), ya que las 
actitudes de los profesionales que afrontan situaciones de impacto pueden modular la calidad de los cuidados que ofrecen (Meier, 2001) e influir en la gestión de su propia calidad de vida interior.

Michael Balint, afirmaba que "el propio médico es la medicina más utilizada en la práctica asistencial". El profesional es la terapia en sí, es la herramienta terapéutica por excelencia" (Balint, 1957). Pero esta herramienta no es imperturbable y no se mantiene siempre estable, por lo que necesita calibrarse y cuidarse de manera permanente.

En esta línea del "medico como herramienta", Novack et al. (1997) hace más de 20 años planteó la necesidad de "calibrar la herramienta terapéutica" y propuso la conciencia personal (self-awareness) como la habilidad que puede mejorar su atención clínica y aumentar la satisfacción con el trabajo, las relaciones personales y consigo mismos.

Según Kearney (Sansó et al., 2015; Kearney et al., 2009) la autoconciencia implica un autoconocimiento y el desarrollo de una conciencia dual o testigo, que permite al clínico atender simultáneamente y evaluar las necesidades del paciente, el entorno de trabajo y su propia experiencia subjetiva.

En 2011 Kearney propone (M. Kearney y Weininger, 2011) un modelo (el autocuidado basado en la autoconciencia), según el cual la autoconciencia sería un factor determinante para establecer una diferencia entre profesionales que "sufren, se contagian y se sienten quemados por el trabajo" y aquellos que en el mismo entorno aprenden, disfrutan y encuentran sentido a su profesión.

En 2015 un estudio pionero en España, se puso a prueba la hipótesis del autocuidado basado en la autoconciencia. En una serie de 387 profesionales de cuidados paliativos, se estudiaron las relaciones entre la competencia profesional para afrontar la muerte, la autoconciencia, la satisfacción de compasión, el estrés traumático secundario, el burnout y el autocuidado (Sansó et al., 2015).

Los resultados confirmaron que el autocuidado y la autoconciencia predijeron de forma positiva la competencia de afrontamiento de los profesionales ante la muerte, y ambas a la vez predijeron positivamente la satisfacción por compasión y negativamente la fatiga por compasión y el burnout.

En resumen, el grado de autoconciencia que puede entrenarse con prácticas orientadas al cultivo de la atención-, en la medida que nos permite una mejor gestión de las emociones, y mayor equilibrio emocional, es un factor que marca una diferencia entre "sufrir o disfrutar en el trabajo" (Stamm, 2012).

Aunque los mecanismos explicativos de cómo funcionan las prácticas de cultivo de la atención son motivo de debate, Vago y Zeidan (2016) proponen que la atención plena conduce a cambios en autoprocesamiento, a través del desarrollo de la autoconciencia (metaconciencia), autorregulación (modulación del comportamiento), y auto-trascendencia (ampliar la perspectiva personal generalmente autocentrada) y ser capaz de verse a sí mismo en un contexto más amplio adquiriendo una actitud pro social (vgr.: compasión).

Hozél et al. (2011) propuso que la atención plena desarrolla sus efectos a través de cambios plásticos de las funciones mentales y cerebrales relacionado con la regulación de la atención, la conciencia del cuerpo (interocepción), la regulación emocional y la toma de autoperspectiva.

Sabemos que el nivel de autoconciencia del profesional cuando es elevado permite, mediante la interocepción, atender y entender 
las emociones que surgen del encuentro $y$ acompañarlas, con ecuanimidad, sabiduría y compasión.

Mantenerse ecuánime pasa por observar y cuidar lo que pensamos y sentimos, y encontrar este espacio interno de seguridad y confianza que no se ve afectado por lo que ocurre en el entorno, y para ello, es fundamental desarrollar un nivel elevado de autoconciencia.

\section{La autoconciencia como plataforma de la presencia y de la regulación emocional}

Si no se dispone de un nivel elevado de conciencia que permita distinguir entre el yo y el otro, el sufrimiento puede ser percibido como una amenaza de la que automáticamente tendemos a huir o a luchar y cuando se experimenta de manera crónica, lo más probable es que el distrés empático genere resultados negativos para la salud (Singer y Klimecki, 2014).

$\mathrm{Si}$ bien el nivel de conciencia es un componente básico para los profesionales sanitarios hay otras características que los define y que no ha sido suficientemente reconocidas en el ámbito académico, nos referimos a la compasión y ala presencia.

La compasión está compuesta por elementos cognitivos, emocionales, intencionales y relacionales que permiten no sólo sostener el sufrimiento ajeno, sino gestionar de forma asertiva las propias emociones.

Es un conjunto de capacidades básicas de los seres humanos para responder al sufrimiento, que se sustenta en los siguientes componentes:

(1) conciencia del sufrimiento (componente cognitivo), (2) preocupación simpática relacionada con ser movido por el sufrimiento (componente afectivo), (3) un deseo de ver el alivio de ese sufrimiento (componente intencional), y (4) la capacidad de respuesta o la disposición para ayudar a aliviar ese sufrimiento (componente motivacional) [38].

A diferencia de los efectos potencialmente perjudiciales del distrés empático, el entrenamiento en compasión promueve la conducta prosocial, y aumenta el afecto positivo y la capacidad de recuperación, desarrollando una mejor gestión de las situaciones estresantes (Singer y Klimecki, 2014; Valk et al., 2017).

Esto abre muchas oportunidades para el desarrollo específico de emociones sociales y motivación, que pueden ser particularmente beneficiosas para las personas que trabajan en profesiones o en entornos estresantes en general (Singer y Klimecki, 2014).

\section{¿Qué es la presencia terapéutica?}

Dice Michael Kearney: "La vida interior del clínico determina la calidad de la presencia que aporta a la cabecera de la cama del enfermo" (M. Kearney y Weininger, 2011).

Significa que el terapeuta lleva todo su ser al encuentro y está completamente presente, a nivel físico, emocional, cognitiva, relacional y espiritual.

Supone estar enraizado en la profundidad de uno mismo y al mismo tiempo conectado al paciente a través de su expresión verbal y no verbal de la experiencia del paciente. Es una forma de estar con el paciente que optimiza la relación terapéutica (Geller y Porges, 2014).

Es una experiencia interna del terapeuta que incluye sentirse:

1) En contacto consigo mismo, enraizado en su propia profundidad, en su esencia que es fuente de serenidad, de paz, de seguridad, con confianza y sin miedo. 
2) Conectado con el sufrimiento de la persona que atiende sin huir, acogiendo todo lo que se da en el encuentro,

3) Percibiendo una perspectiva más amplia y espaciosa, lo que se llama en "expansión", viendo más allá de lo inmediato y con confianza en el proceso que va a seguir desplegándose y

4) Sintiéndose conectado con y al servicio del otro, comprometido con la sanación del paciente.

Esta visión que se fundamenta en la neurofisiología de la comunicación interpersonal, explica la importancia del concepto de" presencia terapéutica" para generar el espacio de seguridad y confianza para el paciente que facilita la corregulación de las emociones.

La teoría Polivagal de Stephen Porges (Porges, 1995; Porges, 2009; Porges S, 2018) nos ayuda a entender "el sistema de conexión social" que hemos desarrollado los mamíferos y más específicamente los humanos. La conexión que se establece en el encuentro terapéutico se sostiene sobre circuitos orgánicos específicos (sistema nervioso autónomo, neurocepción, y sistemas de protección/ defensa desarrollados filogenéticamente), cada vez mejor comprendidos.

Sabemos que estamos dotados de un sistema de "escaneo" constante que explora las señales que recibimos del entorno en búsqueda de amenazas y oportunidades, y son captadas por nuestro sistema fisiológico (neurocepción).

A través de la neurocepción recibimos información subcortical (inicialmente no consciente) que se transmite a través del sistema neurovegetativo, visceral y por vía aferente a través del neumogástrico hacia el SNC. Cuando la información es relevante y se hace consciente hablamos de interocepción (la intuición que nos ofrece información de algo que nos interesa o conviene para nuestra supervivencia).
En la interconexión social hemos desarrollado formas para interpretar estas señales y avisarnos del potencial riesgo, o de que nos encontramos en un entorno de seguridad.

La transmisión de esta información en la interconexión se da a través de las microexpresiones faciales (tono relajado o tenso de la parte superior de los músculos de la cara), el lenguaje corporal de tensión/relajación cierre/apertura y también por el tono de voz, el timbre, la calidez y la prosodia.

Cuando estamos frente a un paciente, utilizamos este sistema de conexión social, a través de los mensajes que nos enviamos mutuamente. En la medida que el paciente se siente amenazado por la enfermedad, su sistema vegetativo está en modo amenaza, (lucha, huida o parálisis). El momento del encuentro terapéutico puede ser visto como una oportunidad de que a través del vínculo con el profesional pueda instaurarse lo que se conoce como co-regulación y el paciente pueda acceder a niveles de serenidad, confianza y paz que le permitan el acceso y la utilización de sus propios recursos, que no percibía en el estado de amenaza.

Para que esto ocurra, el terapeuta debe estar enraizado en su "presencia", es decir, debe ser capaz de trasmitir al paciente su seguridad y confianza, que en la medida que el paciente conecta puede ir apagando su estado de alarma $y$, en la co-regulación que la interacción establece, abrirse a la posibilidad de pasar del estado de amenaza, peligro, tensión a una apertura que le permite acceder a sus recursos internos más apropiados.

La "presencia" es altamente relacional, permitiendo que la serenidad, confianza y paz interior que aporta el terapeuta sea percibido por el paciente, al sentirse escuchado, acogido, entendido y no juzgado, lo que se traduce en una experiencia de sosiego y de calma, que se 
desarrolla a medida que su sistema nervioso se adapta a la presencia tranquilizadora del terapeuta. Las defensas del paciente se suavizan y se retiran los bloqueos, de modo que la expansión natural y la sanación se pueden desplegar. A medida que los dos sistemas nerviosos se conectan, el terapeuta también percibe la experiencia del paciente $y$ manteniéndose en el presente, es capaz de responder en cada momento de forma más adecuada a las necesidades del paciente.

La "presencia" está asociada con la empatía y la compasión, pero es diferente y previa a ambas. Es la plataforma donde éstas pueden expresarse, una vez establecido el contacto y creada la conexión con el paciente, co-creando un espacio relacional que permite que fluya la seguridad y la confianza, escenario que favorece la expresión de la empatía y la compasión.

Desde esta perspectiva se entiende la necesidad de que el propio profesional cultive y desarrolle su equilibrio emocional, su presencia, su capacidad a aporta a la relación terapéutica el máximo potencial de ayuda en forma de "presencia".

\section{6. ¿Qué prácticas son útiles para el autocuidado?}

El nivel de autoconciencia durante el trabajo es un factor crucial para mantener el equilibrio emocional, y que permite al profesional ser consciente de los límites, y condiciones de cada situación, tomar decisiones, y construir oportunidades terapéuticas adaptadas a las posibilidades reales. El concepto de autoconciencia o de presencia terapéutica son el fundamento del autocuidado

Además, el cuidado personal involucra estrategias que ayudan a promover o mantener la salud física, mental, emocional y espiritual; además de garantizar que no se descuiden las necesidades personales $\mathrm{O}$ familiares. EI autocuidado también requiere autorreflexión y conciencia para identificar estresores y apoyos relevantes tanto en el ámbito personal como en el profesional (Mills y Chapman, 2016).

Los profesionales de la salud que se vuelven más conscientes de las influencias de los factores personales en sus actuaciones pueden examinar mejor cómo y por qué toman decisiones. Sus elecciones pueden volverse más consistentes y potencialmente más libres (Kearney y Weininger, 2011). La autoconciencia puede ser un primer paso para estimular cambios actitudinales y conductuales adaptativos en los profesionales de la salud (Kearney y Weininger, 2011).

Cualquier práctica que promueva el cultivo de la atención, cómo el mindfulness, se ha relacionado con la disminución de la tendencia a cargar con las emociones negativas de otros (Cebolla i Martí et al., 2014). Los profesionales con elevado nivel de autoconciencia tienen un mejor autocuidado y una mayor capacidad de implicarse con los pacientes sin experimentar estrés (Cebolla i Martí, et al., 2014). Además, estos profesionales consiguen que el paciente se sienta sentido, convirtiéndose en cuidadores más eficaces (Ashar et al., 2017).

Existe un amplio consenso sobre el hecho de que, junto con la formación avanzada en gestión de emociones, y el desarrollo de la autoconciencia en los sanitarios, a través de la meditación o la práctica espiritual, se asocian a una mejor calidad de vida y a un incremento en la satisfacción profesional o gozo de cuidar en el personal sanitario (Novack et al., 1997; Cole, 1997; Epstein, 1999). 
Tabla 2. Factores de protección del estrés profesional.

- Carga de trabajo sostenible

- Formación en habilidades de comunicación

- Reconocimiento y recompensas adecuadas

- Meditación

- Escritura reflexiva

- Supervisión y tutela

- Desarrollo de habilidades de autoconciencia

- Práctica de actividades de autocuidado

- Actividades de formación continuada

- Participación en investigación

- Uso de rituales

- Programas de reducción de estrés para el equipo basados en mindfulness

- Intervención para el equipo basada en potenciar el sentido del trabajo

- Retiros especializados

- Talleres específicos de autocuidado

- Prácticas integradas en el equipo

Ejemplos de prácticas de autocuidado en el trabajo

Una forma de tratar de conectar periódicamente con la propia autoconciencia, es lo que podemos llamar la práctica de ECP, (estar completamente presente) y supone aprender a parar y resetear la mente: Es sencilla y consiste en:
- Centrarte, (parar), haz 4-5 respiraciones con una larga exhalación. Tomar conciencia de tu cuerpo, de "cómo estás".

- ¿Cómo me siento ahora? ¿Qué tal estoy? ¿tengo alguna tensión en el cuerpo? ¿Tengo alguna preocupación? ¿Hay algo que debería hacer para sentirme mejor? Dejar de ir con "el piloto automático".

- Retomar tu centro, tu capacidad de estar presente y discernir lo que es posible, de lo que no podremos cambiar y aceptarlo.

Al principio puede ser útil de poner "avisos" en el trabajo, alarmas cada varias horas en el teléfono celular para recordarte que necesitas ECP.

Por ejemplo: Sticks o post-its en el entorno para: respirar hondo con largas espiraciones dos o tres veces, recordar centrarse y tomar perspectiva sobre lo que estoy haciendo.

Comentario de un profesional que aplicó esta técnica en su trabajo:

“Las pegatinas de puntos rojos en $\mathrm{mi}$ departamento, fue muy simple, y muy útil; se trata de ponerse al día contigo mismo a veces. La respiración profunda que haces cuando ves un punto. Es como "Está bien, ¿dónde estoy ahora?" Y pensando más sobre dónde estás y desde dónde estás actuando. ¿Qué es lo que te está impulsando en este momento? ¿Por qué es tan importante que tengo que hacerlo ahora? $\mathrm{Y}$ luego tomas un respiro y piensas en ello. Decides si "puedo hacer eso mañana o más tarde hoy y probablemente tenga una mejor manera de hacerlo". Te ayuda a tener una perspectiva y pensar sobre las cosas de una manera más integral."

Ayuda a tomar conciencia y hacer un balance de cómo me siento "y" aliviar la acumulación de estrés. 
¿Cómo nos comportamos cuando estamos estresados? Un ejemplo de un profesional:

Encuentro que en un mal día o en una situación más estresante, estamos frenéticos; no almuerzas hasta las 3 p.m. y no vas al baño todo el día. $Y$ esos pocos segundos en los que simplemente te detienes y piensas: “¿Por qué estoy corriendo como un pollo sin cabeza?

Creo que la tensión física aumenta durante el día. Y si ha habido un problema con un paciente o con otro miembro del personal, estás viviendo eso, y supone más tensión, y creo que solo un par de minutos para respirar, puedes sentir esa sensación apretada, simplemente dejarse ir. Creo que puedo decir que con el tiempo es una forma más saludable de trabajar.

Cuántas veces durante el día exploras ¿cómo me siento ahora? ¿Qué tal estoy? ¿tengo alguna tensión en el cuerpo? ¿Tengo alguna preocupación? ¿Hay algo que debería hacer para sentirme mejor?

Dinámica: en los próximos días... ponte una alarma en el móvil tres veces al día para parar y evaluar cómo te sientes y hacer 5 respiraciones profundas y parar lo que estés haciendo y tomar conciencia de lo que necesitas para estar mejor.

\section{Otro ejemplo de autocuidado:}

Construyendo autocompasión. ¿Cómo te hablas como te tratas cuando hay problemas?, escúchate a ti mismo y veras... que te dices...

La compasión bien entendida empieza por uno mismo, y a menudo nos auto castigamos cuando percibimos que hemos cometido un error. Deberíamos saber que podemos equivocarnos $\sin$ agredirnos $y$ que somos únicamente responsables de aprender de los errores. Es decir, evaluar que deberíamos haber hecho diferente y aprender de ello. Sin autoagredirse sino aceptando la experiencia como oportunidad de aprendizaje.

\section{El trabajo en equipo como factor de protección.}

En cuidados Paliativos hemos aprendido que trabajar en un entorno de alta densidad emocional y enfrentando cotidianamente el sufrimiento, solo es posible trabajando en equipo. Este es no solo una excelente herramienta terapéutica sino además un elemento fundamental en la protección y el autocuidado.

Para que sea así el equipo debe construirse alrededor de los valores compartidos, poniendo en el centro de atención al enfermo y a su familia y trabajando desde el respeto mutuo, y la riqueza que aporta la multidisciplinariedad.

Reconocer en el equipo que nuestro trabajo es duro y estresante y que necesitamos tomar perspectiva sobre ello y considerarlo, ya que negarlo u obviar no ayuda a resolverlo. Compartir en el equipo aspectos de autocuidado, y reservar un espacio en el equipo para evaluar cómo estamos, y lo que necesitamos por ejemplo grupos de apoyo mutuo.

El equipo que se llega a construir como un "nosotros" y da sentido de pertenencia a sus miembros debe reconocer y celebrar su vulnerabilidad en las sesiones $y$ hablar abiertamente de ella. Un grado de madurez del equipo reside en darse permiso para compartir la propia vulnerabilidad y dejar espacios para poder compartir las dificultades o los problemas y cuidarnos. Recientemente la Sociedad española de Cuidados Paliativos a propósito de la pandemia ha elaborado algunos videos 
promoviendo entre otras la función de autocuidado del equipo (Benito, 2020a; Benito, 2020b).

\section{El necesario compromiso del profesional con su autocuidado}

Si queremos ser profesionales centrados en atender personas, deberemos promover el cultivo de nuestra propia humanidad, es decir nuestra capacidad de estar presentes, ser ecuánimes y compasivos, de modo que podamos, en mitad del caos del sufrimiento mantenernos despiertos, íntegros y emocionalmente equilibrados.

Para ello, para se ser buen profesional y estar mentalmente sano y ser más feliz, necesitamos desarrollar nuestro curriculum interior.

Es decir, profundizar en la naturaleza que nos conforma a través del buceo en la propia interioridad, para adquirir los recursos que todos tenemos, y poder afrontar la realidad con mayor grado de conciencia.

Una realidad que incluye las emociones de nuestros pacientes y las propias. Para las que hasta ahora no hemos tenido apenas formación y que, al no saber gestionar, nos están arrastrando a niveles preocupantes de estrés, despersonalización, agotamiento emocional y burnout a los médicos y sanitarios en todo el mundo occidental.

\section{Tabla 3. RESUMEN}

La relación terapéutica se da entre personas, y la vulnerabilidad y la incertidumbre, que acompañan la mayoría de las situaciones clínicas, exigen atender la subjetividad del paciente.

Esta mirada a la persona, y no solo a su patología, exige del profesional habilidades para actuar con compasión y ecuanimidad.

Las emociones pueden contagiarse y la falta de recursos personales del propio profesional para gestionar adecuadamente su impacto, sabemos que es fuente de distrés, y causa de síndrome de estrés post traumático secundario.

Estas habilidades que forman parte del currículum interior, que permiten al profesional sanitario una atención sabia y compasiva, que desarrollamos intuitivamente, y ya están disponibles para incluirlas el ámbito docente.

Las actitudes para poder acompañar y atender de forma eficiente y sana los problemas de nuestros pacientes, es decir los componentes de este curriculum interior son básicamente:

- El cultivo de la dimensión ética que ayude a florecer la intención benevolente, las ganas de ayudar a los que sufren, que subyace en el corazón de cualquier sanitario.

- El cultivo de la atención como base de la propia introspección y autoconocimiento. Es decir, el desarrollo de un grado elevado de autoconciencia.

- Desde aquí poder cultivar la presencia que nos facilita la gestión emocional y la autotrascendencia. 
- Esta es una buena plataforma para ser capaces de establecer una relación terapéutica sana y constructiva.

- La calidad de la comunicación que establecemos con los pacientes y con el equipo, viene determinada por la calidad, profundidad y autenticidad de la comunicación que estableces contigo mismo. Es decir, la comunicación no depende sólo del cómo comunicas, sino sobre todo del "desde donde comunicas".

Retomando la propuesta de William Osler sobre las virtudes de un buen profesional sanitario, ya las resumió para cualquier ser humano un filósofo estoico que nos recordó que

"Debes ser como un promontorio en el mar, contra el cual las olas baten continuamente, pero resiste, y en su cercanía las crecidas olas se tranquilizan y aquietan".

Marco Aurelio

\section{Bibliografía}

Ashar, Y. K., Andrews-Hanna, J. R., Dimidjian, S., y Wager, T. D. (2017). Empathic care and distress: predictive brain markers and dissociable brain systems. Neuron, 94(6), 1263-1273.

Balint, M. (1957). The doctor, his patient and the illness. In London, UK: Tavistock Publications.

Batson, C. D. (2009). These Things Called Empathy: Eight Related but Distinct Phenomena. In The Social Neuroscience of Empathy.

Benito, E. (2020a). EL equipo como herramienta de trabajo y cuidado. SECPAL. https://youtu.be/BCwbeRLnjvU
Benito, E. (2020b). En equipo como grupo de apoyo. https://youtu.be/XSLV-f6DOI0

Calhoun, L. G., y Tedeschi, R. G. (1998). Posttraumatic growth: Future directions. Posttraumatic Growth: Positive Changes in the Aftermath of Crisis, 215-238.

Calhoun, L. G., y Tedeschi, R. G. (1999). Facilitating posttraumatic growth: $A$ clinician's guide. Routledge.

Caroe, T. (2012). Burnout and empathy in primary care. British Journal of General Practice.

Cebolla i Martí, Ausiàs Josep, García-Campayo, J., y Demarzo, M. (2014). Mindfulness y ciencia. Alianza Editorial.

Cole, R. (1997). Meditation in palliative care - A practical tool for self-management. In Palliative Medicine (Vol. 11, Issue 5, pp. 411-413). Arnold, Hodder Headline Plc.

Eisenberg, N., y Eggum, N. D. (2009). Empathic Responding: Sympathy and Personal Distress. In The Social Neuroscience of Empathy.

Epstein, R. M. (1999). Mindful practice. Jama, 282(9), 833-839.

Figley, C. R. (1995). Compassion fatigue as secondary traumatic stress disorder: An overview. In Compassion fatigue: Coping with secondary traumatic stress disorder in those who treat the traumatised.

Geller, S. M., y Porges, S. W. (2014). Therapeutic presence: Neurophysiological mechanisms mediating feeling safe in therapeutic relationships. Journal of Psychotherapy Integration, 24(3), 178-192.

Harrison, R. L., y Westwood, M. J. (2009). 
Preventing vicarious traumatization of mental health therapists: Identifying protective practices. Psychotherapy: Theory, Research, Practice, Training, 46(2), 203.

Hölzel, B. K., Carmody, J., Vangel, M., Congleton, C., Yerramsetti, S. M., Gard, T., y Lazar, S. W. (2011). Mindfulness practice leads to increases in regional brain gray matter density. Psychiatry Research Neuroimaging, 191(1), 36-43.

Kearney, M. K., Weininger, R. B., Vachon, M. L. S., Harrison, R. L., y Mount, B. M. (2009). Self-care of physicians caring for patients at the end of life:"Being connected a key to my survival." JAMA, 301(11), 1155-1164.

Kearney, M., y Weininger, R. (2011). Whole person self-care: Self-care from the inside out. Springer, New York, NY., 109-125.

McCann, I. L., y Pearlman, L. A. (1990). Vicarious traumatization: A framework for understanding the psychological effects of working with victims. Journal of Traumatic Stress, 3(1), 131-149.

Meier, D. E. (2001). The Inner Life of Physicians and Care of the Seriously III. Jama, 286(23), 3007-3014.

Mills, J., y Chapman, M. (2016). Compassion and self-compassion in medicine: Self-care for the caregiver. Australasian Medical Journal, 9(5), 87-91.

Mount, B. M., Boston, P. H., y Cohen, S. R. (2007). Healing connections: on moving from suffering to a sense of well-being. Journal of Pain and Symptom Management, 33(4), 372-388.

Navarro-González, D., Ayechu-Díaz, A., y HuarteLabiano, I. (2015). Prevalencia del síndrome del burnout y factores asociados a dicho síndrome en los profesionales sanitarios de Atención Primaria. Semergen.

Novack, D. H., Suchman, A. L., Clark, W., Epstein, R. M., Najberg, E., y Kaplan, C. (1997). Calibrating the physician: Personal awareness and effective patient care. In Jama (Vol. 278, Issue 6, pp. 502-509). Amer Medical Assoc.

Pearlman, L. A., y Mac Ian, P. S. (1995). Vicarious Traumatization: An Empirical Study of the Effects of Trauma Work on Trauma Therapists. Professional Psychology: Research and Practice, 26(6), 558-565.

Porges, S. W. (2009). The polyvagal theory: New insights into adaptive reactions of the autonomic nervous system. Cleveland Clinic Journal of Medicine.

Porges, S. W. (1995). Orienting in a defensive world: Mammalian modifications of our evolutionary heritage. A Polyvagal Theory. Psychophysiology.

Porges S. (2018). Guía de Bolsillo de la Teoría Polivagal. El poder transformador de sentirse seguro (Elefhteria).

Sansó, N., Galiana, L., Oliver, A., Pascual, A., Sinclair, S., y Benito, E. (2015). Palliative care professionals' inner life: Exploring the relationships among awareness, self-care, and compassion satisfaction and fatigue, burnout, and coping with death. Journal of Pain and Symptom Management, 50(2), 200-207.

Saunders, C. (2011). Velad conmigo. Inspiración Para Una Vida En Cuidados Paliativos. IAHPC Press International Association for Hospice and Palliative Care. Houston TX, USA, 4357.

Shanafelt, T. D., Boone, S., Tan, L., Dyrbye, L. N., 
Sotile, W., Satele, D., West, C. P., Sloan, J., y Oreskovich, M. R. (2012). Burnout and satisfaction with work-life balance among US physicians relative to the general US population. Archives of Internal Medicine, 172(18), 1377-1385.

Singer, T., y Klimecki, O. M. (2014). Empathy and compassion. Current Biology, 24(18), R875R878.

Sir William Osler. (2004). Aequanimitas con otras conferencias a estudiantes de medicina, enfermeras y médicos (Fundación).

Soler, J. K., Yaman, H., Esteva, M., Dobbs, F., Asenova, R. S., Katić, M., Ožvačić, Z., Desgranges, J. P., Moreau, A., y Lionis, C. (2008). Burnout in European family doctors: the EGPRN study. Family Practice, 25(4), 245-265.

\}Stamm, B. H. (2012). Helping the Helpers: Compassion Satisfaction and Compassion Fatigue in Self-Care, Management, and Policy of Suicide Prevention Hotlines.
Resources for Community Suicide Prevention, 1-4.

Tedeschi, R. G., y Calhoun, L. G. (1995). Trauma and transformation. Sage.

Vago, D. R., y Zeidan, F. (2016). The brain on silent: mind wandering, mindful awareness, and states of mental tranquility. Annals of the New York Academy of Sciences, 1373(1), 96-113.

Valk, S. L., Bernhardt, B. C., Trautwein, F.-M., Böckler, A., Kanske, P., Guizard, N., Collins, D. L., Singer, T., Hombrados-mendieta, I., Singer, T., Klimecki, O. M., Gupta, R., Singh, N., y Kumar, R. (2017). Structural plasticity of the social brain: Differential change after socio-affective and cognitive mental training. Science Advances, 3(10), e1700489.

Whintrobe M, Thorn G, Adams R, Bennett I, H. B. (1973). Principios de Medicina Interna. In Prensa Médica Mexicana: Vol. 4 a edición. 